\title{
La protección en la jurisdicción penal internacional del derecho a la cultura: los crímenes internacionales de destrucción del patrimonio cultural \\ The protection under international criminal jurisdiction of the right to culture: internacional crimes regarding destruction of cultural heritage
}

\author{
Maria Torres Pérez \\ Departamento de Derecho Internacional “Miaja de la Muela” \\ Facultat de Dret \\ Universitat de València
}

\section{RESUMEN.}

El presente artículo pretender dar una visión global de la protección del derecho a la cultura a través del derecho internacional penal y en concreto, a través de la tipificación de los crímenes de destrucción del patrimonio cultural. La persecución de tales delitos internacionales ha seguido la senda de otros crímenes en derecho internacional penal, englobándose en un primer momento entre los crímenes de guerra para de forma posterior superar dicha tipificación y pasar a ser tratado como crímen de lesa humanidad de persecución e incluso, como indicio de una conducta genocida. En el presente contexto internacional, la denegación del derecho a la cultura como método de guerra es innegable en los conflictos protagonizados por los nuevos actores internacionales por lo que la solución final del derecho internacional penal debe progresar para erigirse en un método de protección privilegiado.

\section{PALABRAS CLAVE.}

Derecho Penal Internacional, destrucción del patrimonio cultural, persecución, tribunales penales internacionales.

\section{ABSTRACT.}

The present article intends to give a global vision of the protection of the right to culture through international criminal law and in particular, through the tipification of crimes of destruction of cultural heritage. The prosecution of such international crimes has followed the path of other crimes in international criminal law, initially included in the type of war crimes and later overcoming such tipification in order to be treated as a crime against humanity of persecution and even, as indications of a genocidal behavior. In the present international context, the denial of the right to culture as a method of warfare is undeniable in conflicts involving new international actors, so the final solution of international criminal law must progress to become a privileged method of protection.

\section{KEY WORDS.}

International Criminal Law, destruction of cultural heritage, persecution, internacional criminal tribunals.

CEED Número 39 Publicación actas Congreso Internacional 70 Aniversario Declaración Universal de Derechos 


\section{Introducción}

El derecho a la cultura reconocido en los Pactos de Derechos Humanos de 1966 engloba en su faceta más colectiva el derecho a disfrutar del patrimonio cultural, compendio de la historia presente y futura de cualquier grupo humano desde su origen. Así, la Comunidad Internacional ha ido entendiendo que dicho patrimonio cultural merece de protección tanto en tiempos de paz como en tiempos de conflicto armado, ya que el mismo forma parte del patrimonio global de la humanidad y como tal el derecho internacional debe interesarse en su mantenimiento.

Los derechos culturales, como afirma el Comité de Derechos Económicos, Sociales y Culturales, en su Observación General núm. 21 (2009), relativa al derecho de toda persona a participar en la vida cultural:

“(...) protegen los derechos de todas las personas, individualmente y en comunidad con otros, así como de grupos de personas, para desarrollar y expresar su humanidad, su visión del mundo y el significado que dan a su existencia y desarrollo mediante, entre otras cosas, valores, creencias, convicciones, idiomas, los conocimientos y las artes, las instituciones y las formas de vida. Puede considerarse también que protegen el acceso al patrimonio cultural y a recursos que permiten que esos procesos de identificación y desarrollo tengan lugar"1.

La inclusión de la destrucción del patrimonio cultural entre las conductas tipificadas en el Estatuto de Roma de la Corte Penal Internacional ${ }^{2}$ como crímenes internacionales es un reflejo tanto de la labor codificadora llevada a cabo por la Comunidad Internacional en defensa del patrimonio cultural como de la jurisprudencia internacional que ha ido desarrollándose en materia de protección de dicho patrimonio, al considerar dicha protección un objetivo común de los Estados ${ }^{3}$ íntimamente ligado al objetivo global de mantenimiento de la paz y seguridad internacionales ${ }^{4}$.

\footnotetext{
${ }^{1} \mathrm{~A} / \mathrm{HRC} / 14 / 36$, párr. 9, y A/67/287, párr. 7

${ }^{2}$ Estatuto de Roma de la Corte Penal Internacional, hecho en Roma el 17 de julio de 1998. BOE núm. 126, de 27 de mayo de 2002 . En adelante, CPI.

${ }^{3}$ Según afirmó la UNESCO en el Amicus curiae presentado ante la Corte Penal Internacional (en adelante, CPI) en el asunto The Prosecutor v. Ahmad Al Faqi Al Mahdi, ICC-01/12-01/15, "La pérdida del patrimonio durante tiempos de conflicto puede arrebatar a una comunidad su identidad y su memoria. Aquellos que destruyen el patrimonio cultural buscan debilitar los cimientos de la cohesión nacional a través de la propagación del miedo y del odio, debilitando la resistencia y rompiendo los lazos que unen a los individuos y a los grupos. La UNESCO cree firmemente que tal "limpieza cultural" constituye un asunto de seguridad. Proteger la cultura es un valor
}

CEED Número 39 Publicación actas Congreso Internacional 70 Aniversario Declaración Universal de Derechos 
La destrucción del patrimonio cultural se incluye, a saber, en diversas de las conductas tipificadas como crímenes bajo la jurisdicción de la CPI. En primer lugar, se ha visto tipificada como crímenes del artículo 8 (crímenes de guerra) derivados de las violaciones graves de los Convenios de Ginebra de 1949 (la destrucción y la apropiación de bienes, no justificadas por necesidades militares, y efectuadas a gran escala, ilícita y arbitrariamente) o de otras violaciones graves de las leyes y usos aplicables en los conflictos armados internacionales o no internacionales (dirigir intencionalmente ataques contra edificios dedicados a la religión, la educación, las artes, las ciencias o la beneficencia, los monumentos históricos, los hospitales y los lugares en que se agrupa a enfermos y heridos, siempre que no sean objetivos militares).

Pero tal tipificación como crimen de guerra no agota las posibilidades de persecución penal de los actos de destrucción del patrimonio cultural, ya que en segundo lugar y recogiendo la jurisprudencia internacional hasta el momento, el Estatuto de la CPI permite su tipificación como crimen de lesa humanidad de persecución definido en el art. 7.1.h) y 7.2 del propio Estatuto.

Es esta configuración novedosa la que permite tener en cuenta toda una serie de situaciones que quedan fuera de las situaciones de conflictos armados ${ }^{5}$. A saber, las situaciones en las que la destrucción se produce durante ataques sistemáticos y generalizados contra la población civil que no alcanzan la categoría de conflicto armado y que se llevan a cabo con la especial motivación de privar al grupo de su identidad mediante la eliminación de uno de sus derechos fundamentales; en este caso, el derecho fundamental a su desarrollo cultural reconocido tanto en el Pacto Internacional de Derechos Civiles y Políticos como en el Pacto Internacional de Derechos Económicos, Sociales y Culturales.

esencial de la comunidad internacional que no puede separarse de la protección de la vida humana. (...)”, (la traducción es nuestra). El texto original está disponible en https://www.icc-cpi.int/CourtRecords/CR2016 25595.PDF, consultada el 5 de abril de 2018.

4 S/RES/2347 (2017), de 24 de marzo de 2017, Resolución 2347 (2017) aprobada por el Consejo de Seguridad en su 7907a sesión, celebrada el 24 de marzo de 2017, disponible en https://www.un.org/es/comun/docs/?symbol=S/RES/2347(2017).

5 Tal y como señalan algunos autores, el patrimonio cultural durante una situación de conflicto armado se enfrenta a cuatro peligros fácilmente determinables. En primer lugar, la posibilidad de destrucción por acciones intencionales de los combatientes; en segundo lugar, el daño colateral que puede acontecer al atacarse objetivos militares legítimos; en tercer lugar, el daño derivado de la falta de conocimiento o ignorancia de la presencia de patrimonio cultural; y, por último, el daño que deriva del saqueo y robo de objetos culturales. En este sentido, véase: Véase GERTENBLITH, P., "Archaeology in the Context of War: Legal Frameworks for Protecting Cultural Heritage during Armed Conflict”, Archaeologies: Journal of World Archaeological Congress, 5/1, 2009, pp. 18-31. 
Y es esta configuración la que se presenta como especialmente interesante ante los conflictos armados recientes en los que la Comunidad Internacional se ha visto desbordada por la aparición de nuevos actores internacionales que no se consideran ligados por los textos convencionales dedicados a la protección del patrimonio cultural. Entre estos nuevos actores se encuentran los grupos terroristas, que no sólo destruyen el patrimonio cultural a través del saqueo y el contrabando de sus bienes como fuente de financiación, sino que incluso convierten la destrucción de dicho patrimonio en parte de su estrategia para alimentar los conflictos y acabar con la diversidad cultural de las zonas que se ven acosadas a través de tal estrategia de guerra ${ }^{6}$.

Los ataques sistemáticos y generalizados que configuran el crimen de lesa humanidad en el caso de la CPI, son definitorios del propio actuar del terrorismo, por lo que la extensión de responsabilidad a estos nuevos actores podría producirse teniendo en cuenta la consideración de los crímenes de lesa humanidad como normas de ius cogens.

Las primeras sentencias internacionales en el ámbito penal sobre la destrucción del patrimonio cultural como crimen de lesa humanidad de persecución, como señalábamos, fueron las emitidas por el Tribunal Penal Internacional para la Antigua Yugoslavia entre los años 1993 a $2017^{7}$. Otros tribunales internacionales penales ad hoc o mixtos también han incluido la destrucción del patrimonio cultural entre los crímenes bajo su jurisdicción, pero sin duda, fue el TIPY el que de forma más extensa trató y dictaminó sobre la misma, iniciando un camino en el que la protección se extiende a la última ratio del derecho penal internacional.

\footnotetext{
${ }^{6}$ Tal y como señalan numerosos autores, los conflictos recientes que han puesto en peligro al patrimonio cultural están íntimamente ligados con luchas por la identidad nacional o religiosa, en los que acaban participando numerosos actores de diverso carácter y agenda, y en los que la destrucción del patrimonio cultural se convierte en un objetivo en sí mismo. Véase, VAN DER AUWERA, S., "Contemporary Conflict, Nationalism, and the Destruction of Cultural Property during Armed Conflict: A Theoretical Framework", Journal of Conflict Archaeology, 7/1, 2012, pp. 49-65.

${ }^{7}$ El Tribunal Internacional Penal para la Antigua Yugoslavia (en adelante, TIPY) concluyó su mandato el pasado 31 de diciembre de 2017, haciéndose cargo de los procesos abiertos el United Nations Mechanism for Internacional Criminal Tribunals (en adelante, MICT). Sobre la protección del patrimonio cultural por el TIPY, véase: LOSTAL BECERRIL, M., La protección de bienes culturales en el Tribunal Internacional Penal para la Ex Yugoslavia, REEI, 24, 2012, 25 págs. Disponible en http://www.reei.org/index.php/revista/num24/notas/proteccion-bienes-culturales-tribunal-penal-internacional-para-ex-yugoslavia.
}

CEED Número 39 Publicación actas Congreso Internacional 70 Aniversario Declaración Universal de Derechos 
Como hemos señalado, la antorcha en tal protección se ha trasladado a la CPI por lo que el estudio y conocimiento del anterior cuerpo jurisprudencial es sin duda necesario para poder apoyar y comprender las actuales y futuras sentencias que pudiera dictar la CPI.

\section{La tipificación penal de las conductas atentatorias contra el patrimonio cultural en el Estatuto y la jurisprudencia del TIPY.}

Las violaciones sufridas por el patrimonio cultural durante las guerras de los Balcanes enfrentaron a la Comunidad internacional a imágenes ya olvidadas de destrucción en el territorio del viejo continente ${ }^{8}$. Tales destrucciones conllevaron una reforma del cuerpo legal humanitario destinado a la protección de los bienes culturales, a saber, la Convención de La Haya de $1954^{9}$ y su primer protocolo ${ }^{10}$ que se habían demostrado ineficaces a la hora de implementarse durante el conflicto. El segundo Protocolo a la Convención ${ }^{11}$ vio la luz en 1999, no pudiendo recogerse expresamente ni en el Estatuto del TIPY ni en el Estatuto de la CPI pero suponiendo un nuevo enfoque que merecerá de valoración por la jurisprudencia posterior de la CPI o en futuras reformas del Estatuto o en el documento de los Elementos de los Crímenes.

El Estatuto del TIPY ${ }^{12}$, recogiendo el derecho internacional consuetudinario en la materia con el fin de respetar los principios fundamentales del nullum crimen sine lege y de irretroactividad de las sanciones penales, preveía la tipificación de las conductas de destrucción del patrimonio cultural bajo el epígrafe dedicado a los crímenes de guerra, ya como violaciones a los Convenios de Ginebra de 1949 (artículo 2 d) "destrucción o apropiación de bienes no justificados por necesidades militares y llevadas a cabo en gran escala y en forma ilícita y arbitraria") ya como violaciones de las leyes y usos de la guerra

\footnotetext{
${ }^{8}$ Sobre el conflicto armado de la antigua Yugoslavia y la protección del patrimonio cultural, véase BADENES CASINO, M. (2005), La protección de los bienes culturales durante los conflictos armados. Especial referencia al conflicto armado en el territorio de la antigua Yugoslavia, Valencia, Universitat de València, 2005, 162 pp.

9 Convención para la Protección de los Bienes Culturales en caso de Conflicto Armado y Reglamento para la aplicación de la Convención, de 14 de mayo de 1954. BOE núm. 282, de 24 de noviembre de 1960. A fecha de redacción del presente artículo, 131 Estados son partes de la Convención.

${ }^{10}$ Protocolo a la Convención para la protección de los bienes culturales en caso de conflicto armado, de 14 de mayo de 1954 . BOE núm. 178, de 25 de julio de 1992. A fecha de redacción del presente artículo, 109 Estados son partes del Primer Protocolo.

${ }^{11}$ Segundo Protocolo a la Convención para la protección de los bienes culturales en caso de conflicto armado, de 26 de marzo de 1999. BOE núm. 77, de 30 de marzo de 2004. A fecha de redacción del presente artículo, únicamente 78 se encuentran vinculados por el Segundo Protocolo.

${ }^{12}$ Estatuto del TIPY, disponible en http://www.icty.org/x/file/Legal\%20Library/Statute/statute sept09 en.pdf.
}

CEED Número 39 Publicación actas Congreso Internacional 70 Aniversario Declaración Universal de Derechos 
(artículo 3d) "la apropiación o destrucción de instituciones consagradas al culto religioso, la beneficencia y la educación o a las artes y las ciencias, monumentos históricos u obras de arte y científicas, o los daños deliberados a éstos”).

En virtud de tal previsión, la Oficina del fiscal acusó de tales cargos a 17 de los procesados ante el TIPY $^{13}$. En todos los asuntos, el cargo basado en la destrucción de patrimonio cultural se sumaba a otros cargos en el acta de acusación, por lo que nunca fue base única de ninguna de las condenas del TIPY.

La labor iniciada por la fiscalía permitió al Tribunal precisar los elementos definitorios de los crímenes de guerra contra el patrimonio cultural en el derecho internacional consuetudinario, ya que:

"the Chamber is satisfied that Article 3(d) of the Statute is a rule of international humanitarian law which not only reflects customary international law but is applicable to both international and noninternational armed conflict. (...) Further, the Kordic Trial Judgement held that while this offence overlaps to a certain extent with the offence of unlawful attacks on civilians objects, when the acts in question are directed against cultural heritage, the provision of Article 3(d) is lex specialis. "14.

\footnotetext{
${ }^{13}$ Los acusados bajo el cargo de crímenes de guerra o violaciones de las leyes y usos de guerra fueron: Tihomir Blaškić (Asunto “Lašva Valley" (IT-95-14), The Prosecutor v. Tihomir Blaškić. Toda la documentación sobre el caso se encuentra disponible en http://www.icty.org/case/blaskic/4.); Radoslav Brđain (Asunto "Krajina" (IT-99-36), The Prosecutor v. Radoslav Brđain. Toda la documentación sobre el caso se encuentra disponible en http://www.icty.org/case/brdanin/4); Goran Hadžić (Asunto IT-04-756, The Prosecutor v. Goran Hadžić. Toda la documentación sobre el caso se encuentra disponible en http://www.icty.org/case/hadzic/4.); Enver Hadžihasanović (Asunto "Hadžihasanović \& Kubura" (IT-01-47), The Prosecutor v. Enver Hadžihasanović and Amir Kubura. Toda la documentación sobre el caso se encuentra disponible en http://www.icty.org/case/hadzihasanovic kubura/4); Miodrag Jokić (Asunto "Dubrovnik" (IT-01-42/1), The Prosecutor v. Miodrag Jokić. Toda la documentación sobre el caso se encuentra disponible en http://www.icty.org/case/miodrag jokic/4); Dario Kordić y Mario Čerkez (Asunto “Lašva Valley” (IT-95-14/2), The Prosecutor v. Dario Kordić y Mario Čerkez. Toda la documentación sobre el caso se encuentra disponible en http://www.icty.org/case/kordic cerkez/4); Milan Martić (Asunto "RSK" (IT-95-11), The Prosecutor v. Milan Martić. Toda la documentación sobre el caso se encuentra disponible en http://www.icty.org/case/martic/4); Slobodan Milošević (Asunto “Kosovo, Croatia \& Bosnia”(IT-02-54), The Prosecutor v. Slobodan Milošević. Toda la documentación sobre el caso se encuentra disponible en http://www.icty.org/case/slobodan_milosevic/4); Jadranko Prlić, Bruno Stojić, Slobodan Praljak, Milivoj Petković, Valentin Ćorić y Berislav Pušić (Asunto "Prlić et al." (IT-04-74), The Prosecutor v. Jadranko Prlić, Bruno Stojić, Slobodan Praljak, Milivoj Petković, Valentin Ćrić \& Berislav Pušić. Toda la documentación sobre el caso se encuentra disponible en http://www.icty.org/case/prlic/4); Pavle Strugar (Asunto "Dubrovnik" (IT-01-42), The Prosecutor v. Pavle Strugar. Toda la documentación sobre el caso se encuentra disponible en http://www.icty.org/case/strugar/4); y Momir Talić (Asunto "Krajina” (IT-99-36/1), The Prosecutor v. Momir Talić. Toda la documentación sobre el caso se encuentra disponible en http://www.icty.org/case/talic/4).
}

${ }^{14}$ Párr. 230 y 302 de la sentencia de instancia en el asunto Asunto "Dubrovnik" (IT-01-42), The Prosecutor v. Pavle Strugar. 
Como requisito general, para ser considerado como un crimen de guerra, la destrucción del patrimonio debe cometerse, dejando de lado lo obvia que puede resultar la precisión, en el contexto de un conflicto armado declarado. Sin embargo, en este caso, la categorización de tal conflicto armado como interno o de carácter internacional no supondrá ningún cambio en la categoría del crimen, castigándose del mismo modo y con la misma fuerza cualquier tipo de destrucción producida sin distinción del conflicto $^{15}$.

Tras establecer la existencia del conflicto armado, los elementos del tipo penal específico se sistematizaron en numerosas sentencias, resultando especialmente clarificadora la dictada en el asunto Dubrovnik. En ella, la Sala consideró que el derecho internacional consuetudinario definía el crimen de guerra de destrucción de bienes culturales a través de los siguientes elementos:

"In view of the above, the definition established by the jurisprudence of the Tribunal appears to reflect the position under customary international law. For the purposes of this case, an act will fulfil the elements of the crime of destruction or wilful damage of cultural property, within the meaning of Article $3(d)$ of the Statute and in so far as that provision relates to cultural property, if: (i) it has caused damage or destruction to property which constitutes the cultural or spiritual heritage of peoples; (ii) the damaged or destroyed property was not used for military purposes at the time when the acts of hostility directed against these objects took place; and (iii) the act was carried out with the intent to damage or destroy the property in question" 16.

La Oficina del Fiscal, sin embargo, consideró que tal configuración no permitía perseguir de forma completa los crímenes cometidos contra el patrimonio cultural en el conflicto de la Antigua Yugoslavia. Junto con ello, se entendió que el derecho internacional consuetudinario había evolucionado de tal modo que la protección penal abarcaba otras situaciones. Así, la fiscalía se encargó de incluir actos de destrucción del patrimonio cultural como elementos indiciarios de otros crímenes bajo la jurisdicción de la Corte; a saber, como indiciario de un crimen de lesa humanidad de

\footnotetext{
15 Según señala MERON, la inclusión en el artículo 3d como violación de las leyes y usos de guerra era la más adecuada, ya que permitía la persecución del crimen tanto si el conflicto armado era internacional como si no revestía tal carácter. De ese modo, la Oficina del Fiscal dirigió sus esfuerzos a incluirlo en tal previsión. Véase, MERON, T., "The Protection of Cultural Property in the Event of Armed Conflict within the Case-law of the International Criminal Tribunal for the Former Yugoslavia", Museum International, 57/4, 2005, pp. 41-60.

${ }^{16}$ Párr. 312 de la sentencia de instancia en el asunto Asunto “Dubrovnik” (IT-01-42), The Prosecutor v. Pavle Strugar.
}

CEED Número 39 Publicación actas Congreso Internacional 70 Aniversario Declaración Universal de Derechos 
persecución $^{17}$ e, incluso, como parte de la estrategia de destrucción del grupo que debe existir en el crimen de genocidio.

El tipo penal del crimen de lesa humanidad de persecución, desde las primeras sentencias emitidas por las Salas de instancia y apelación, se reconoce como un tipo complejo, que puede asumir diversas formas criminales que deberían definirse a través de los instrumentos internacionales sobre protección de los derechos humanos tanto de valor consuetudinario como convencional, considerando como persecución, con carácter general, cualquier acto u omisión que de hecho discrimine y deniegue o infrinja un derecho humano fundamental siempre que sean actos de igual gravedad a los recogidos en el listado de crímenes de lesa humanidad del Estatuto ${ }^{18}$.

Así, la Sala de instancia en el asunto "Lasva Valley” (IT-95-14) reconocía que:

“(...) «Persecution» may asume several different criminal forms. The indictment against General Blaškić specifies physical and mental injury (murders and other forms of bodily harm including the use of civilians as human shields and forcing them to dig trenches), infringements upon individual freedom (arbitrary arrest and detention and forcible transfer of civilians) and attacks against property (destruction and plunder of property), all directed against the «Bosnian Muslim civilian population».

Although the Statute of the Nuremberg Tribunal and those of the Tribunals for the former Yugoslavia and Rwanda all sanction persecutions on political, racial and religious grounds under crimes against humanity, none defines this sub-characterisation or states which forms it may take. The Trial Chamber

\footnotetext{
${ }^{17}$ El Fiscal del TIPY incluyó actos de destrucción del patrimonio cultural entre las bases del crimen de lesa humanidad de persecución en las actas de acusación de: Milan Babić (Asunto "RSK" (IT-03-72), The Prosecutor v. Milan Babić. Toda la documentación sobre el caso se encuentra disponible en http://www.icty.org/case/babic/4); Tihomir Blaškić (Asunto "Lašva Valley” (IT-95-14), The Prosecutor v. Tihomir Blaškić. Toda la documentación sobre el caso se encuentra disponible en http://www.icty.org/case/blaskic/4.); Dario Kordic y Mario Cerkez (Asunto “Lašva Valley” (IT-95-14/2), The Prosecutor v. Dario Kordic \& Mario Cerkez. Toda la documentación sobre el caso se encuentra disponible en http://www.icty.org/case/kordic_cerkez/4); Bobetko (Asunto "Medak Pocket" (IT-02-62), The Prosecutor v. Janko Bobetko. Toda la documentación sobre el caso se encuentra disponible en http://www.icty.org/case/bobetko/4.

${ }^{18}$ Según afirmó la Sala de apelación en su sentencia de 3 de abril de 2007 en el asunto "Krajina" (IT-99-36), The Prosecutor v. Radoslav Brđain, pár. 296, "it is settled jurisprudence that the crime of persecution can include acts which are listed as crimes under Article 5 of the Statute, or under other articles of the Statute, as well as acts which are not listed in the Statute. The Appeals Chamber recalls that acts underlying persecutions under Article 5(h) of the Statute need not necessarily be considered a crime in international law. For the acts not enumerated as a crime in the Statute to amount to the crime of persecution pursuant to Article 5(h) of the Statute, they must be of equal gravity to the crimes listed in Article 5 of the Statute, whether considered in isolation or in conjunction with other acts".
}

CEED Número 39 Publicación actas Congreso Internacional 70 Aniversario Declaración Universal de Derechos 
will therefore refer to customary international law to determine whether the violations covered in the indictment may constitute persecution and under what condition they may be characterised as such" 19.

Así, junto a los elementos comunes de los crímenes de lesa humanidad - a saber, la existencia de una ataque generalizado o sistemático contra la población civil y el necesario mens rea- cualquier tipo de persecución debe cumplir tres requisitos adicionales:

"In its definition of the actus reus of persecution, the Trial Chamber in Kupreškic set forth a four-part test in which an act of persecution is constituted by (1) a gross or blatant denial, (2) on discriminatory grounds, (3) of a fundamental right, laid down in international customary or treaty law, (4) reaching the same level of gravity as the other crimes against humanity enumerated in Article 5 of the Statute.245 The Trial Chamber finds that acts which meet the four criteria set out above, as well as the general requirements applicable to all crimes against humanity, may qualify as persecution, without violating the principle of legality. "20.

Concluyendo que:

"This act, when perpetrated with the requisite discriminatory intent, amounts to an attack on the very religious identity of a people. As such, it manifests a nearly pure expression of the notion of "crimes against humanity", for all of humanity is indeed injured by the destruction of a unique religious culture and its concomitant cultural objects. The Trial Chamber therefore finds that the destruction and wilful damage of institutions dedicated to Muslim religion or education, coupled with the requisite discriminatory intent, may amount to an act of persecution" 21.

\footnotetext{
${ }^{19}$ Párr. 218-219 de la Sentencia de Instancia en el asunto “Lašva Valley” (IT-95-14), The Prosecutor v. Tihomir Blaškić, de 3 de marzo de 2000 .

${ }^{20}$ Párr.195 de la sentencia de instancia en el asunto The Prosecutor v. Dario Kordic \& Mario Cerkez, Asunto "Lašva Valley" (IT-9514/2).

${ }^{21}$ Ídem, párr. 207.
}

CEED Número 39 Publicación actas Congreso Internacional 70 Aniversario Declaración Universal de Derechos 


\section{La inclusión de las conductas atentatorias contra el patrimonio cultural en el Estatuto de la CPI.}

La firma del Estatuto de la CPI en 1998 tampoco permitió incluir las mejoras que se recogerían en el Segundo Protocolo a la Convención de 1954, pero sí que recogió la jurisprudencia mencionada en el apartado anterior, reconociendo la labor llevada a cabo por el TIPY.

Así, el Estatuto de la CPI recoge la destrucción del patrimonio cultural en diversas de las conductas tipificadas como crímenes bajo su jurisdicción. Por un lado, puede ser tipificado como entre los crímenes del artículo 8 (crímenes de guerra) derivados de las violaciones graves de los Convenios de Ginebra de 1949 (la destrucción y la apropiación de bienes, no justificadas por necesidades militares, y efectuadas a gran escala, ilícita y arbitrariamente) o de otras violaciones graves de las leyes y usos aplicables en los conflictos armados internacionales o no internacionales (dirigir intencionalmente ataques contra edificios dedicados a la religión, la educación, las artes, las ciencias o la beneficencia, los monumentos históricos, los hospitales y los lugares en que se agrupa a enfermos y heridos, siempre que no sean objetivos militares). Y, por otro lado, como crimen de lesa humanidad de persecución definido en el art. 7.1.h) y 7.2 del propio Estatuto.

Las definiciones que se contemplan en el Estatuto se completan mediante el documento de Elementos de los Crímenes $^{22}$, encargado de conformidad con el artículo 9 del Estatuto, de ayudar a la Corte a interpretar y a aplicar los artículos 6, 7 y 8 en forma compatible con el Estatuto.

Respecto a los crímenes del artículo 8, el DEC contempla toda una serie de requisitos generales respecto al autor (lanzar el ataque, la existencia de conflicto armado y el conocimiento del autor de la existencia del mismo) y dos requisitos principales sobre los bienes. En primer lugar, la categorización del bien como protegido y, en segundo lugar, que el bien no pueda ser considerado como objetivo militar. Tales precisiones remiten al derecho internacional humanitario consuetudinario recogido en los Convenios de Ginebra o en las normas dedicadas a la protección del patrimonio en tiempos de conflicto armado entre las que figuran los Convenios de La Haya de 1899 y 1907 y, de manera destacada, la Convención de La Haya de 1954 y sus Protocolos de 1954 y 1999.

22 En adelante, DEC. El documento en castellano se encuentra disponible en https://asp.icccpi.int/iccdocs/asp docs/Publications/Compendium/ElementsOfCrime-SPA.pdf, visitada el 22 de noviembre de 2018.

CEED Número 39 Publicación actas Congreso Internacional 70 Aniversario Declaración Universal de Derechos 
En virtud de tal previsión, la CPI ha sido quien ha logrado la primera condena a nivel internacional por un crimen de guerra de destrucción del patrimonio cultural de forma individual, no ligada a la comisión de otros crímenes tipificados en el Estatuto. Así, el pasado 27 de septiembre de 2016, la CPI dictaba su primera condena en el asunto The Prosecutor v. Ahmad Al Faqi Al Mahdi ${ }^{23}$ en la que la Corte defiende la gravedad de estos crímenes, a pesar de que no se acusara al detenido de crímenes contra las personas, basada en la importancia del patrimonio no sólo para los habitantes de Mali sino para toda la Comunidad Internacional, y en el motivo de su comisión. Así, afirma la Corte:

"Thus, the Chamber considers that the fact that the targeted buildings were not only religious buildings but had also a symbolic and emotional value for the inhabitants of Timbuktu is relevant in assessing the gravity of the crime committed.

Furthermore, all the sites but one (the Sheikh Mohamed Mahmoud Al Arawani Mausoleum) were UNESCO World Heritage sites and, as such, their attack appears to be of particular gravity as their destruction does not only affect the direct victims of the crimes, namely the faithful and inhabitants of Timbuktu, but also people throughout Mali and the international community. (...)

Lastly, the Chamber notes that the crime was committed for religious motives. (...). The Chamber considers that the discriminatory religious motive invoked for the destruction of the sites is undoubtedly relevant to its assessment of the gravity of the crime.

The Chamber concludes that the crime for which Mr Al Mahdi is convicted is of significant gravity". ${ }^{24}$

Por lo que se refiere al crimen de lesa humanidad de persecución, y según contempla el art. 7.2:

g) Por «persecución» se entenderá la privación intencional y grave de derechos fundamentales en contravención del derecho internacional en razón de la identidad del grupo o de la colectividad.

\footnotetext{
${ }^{23}$ Op. cit. nota 7.

${ }^{24}$ Párr. 79 a 82 del Public Judgment and Sentence disponible en https://www.icc-cpi.int/CourtRecords/CR2016 07244.PDF.
}

CEFD Número 39 Publicación actas Congreso Internacional 70 Aniversario Declaración Universal de Derechos 
Tal previsión se completa con el DEC que establece la necesidad de que la conducta criminal se dirija contra las víctimas por razón de su identidad de grupo o colectividad y por los motivos políticos, raciales, nacionales, étnicos, culturales, religiosos o de género, según la definición del párrafo 3 del artículo 7 del Estatuto, o por otros motivos universalmente reconocidos como inaceptables con arreglo al derecho internacional. En cualquier caso, tal conducta deberá cometerse en relación con cualquiera de los actos señalados en el párrafo 1 del artículo 7 del Estatuto o con cualquier crimen de la competencia de la Corte y como parte de un ataque generalizado o sistemático dirigido contra una población civil, del que el autor debe tener conocimiento.

La restricción contenida en el DEC hace que el crimen de lesa humanidad de persecución por destrucción del patrimonio cultural no pueda ser un cargo único en un futuro asunto ante la CPI, ya que se ha considerado, a nuestro modo de ver de forma injusta, que en su misión de garante de la legalidad penal internacional para los más graves crímenes perseguir los crímenes de lesa humanidad de persecución no acompañados de otras violaciones sería de menor importancia. Tal consideración parece olvidar que, en muchas ocasiones, los crímenes de persecución son los primeros en aparecer, antes de que se produzcan las matanzas o violaciones en masa y que los crímenes de lesa humanidad protegen precisamente a una parte de la población civil que habitualmente se encuentra abandonada por los poderes públicos, que incluso coadyuvan a tal persecución.

\section{Otros tribunales penales internacionales o mixtos.}

La labor del TIPY ha sido recogida por otros tribunales internacionales ad hoc e incluso por los tribunales denominados como híbridos ${ }^{25}$, entre los que cabe destacar de forma especial a las Salas Extraordinarias de Camboya ${ }^{26}$ con competencia para juzgar a los líderes de la Kampuchea Democrática por los crímenes cometidos entre el 17 de abril de 1975 al 6 de enero de 1979, tanto en base al derecho

\footnotetext{
${ }^{25}$ Con el término "híbridos", la doctrina se refiere a los Tribunales mixtos que se han ido generando desde la constitución del TIPY en los que la jurisdicción y la ley aplicada es tanto internacional como interna. En este grupo se incluyen las Salas Extraordinarias de Camboya o el Tribunal Especial para Sierra Leona. Sobre los tribunales híbridos, véase:

26 El Estatuto de las Salas Extraordinarias de Camboya está disponible en http://www.legaltools.org/uploads/tx_ltpdb/Cambodia_LawontheEstablishmentofExtraordinaryChambers_10-08-2001_ E _ 06.htm. Sobre las Salas Extraordinarias, véase: LAMBOURNE, W., "The Khmer Rouge Tribunal: Justice for Genocide in Cambodia?", en Law and Society Association Australia and New Zealand (LSAANZ) Conference, 'W(h)ither Human Rights', 2008, pp. 1-11; KLEIN, K. M., "Bringing the Khmer Rouge to Justice: The Challenges and Risks Facing the Joint Tribunal in Cambodia", Northwestern University Journal of International Human Rights, 4(3), 2005, pp. 549-566.
}

CEFD Número 39 Publicación actas Congreso Internacional 70 Aniversario Declaración Universal de Derechos 
camboyano como al derecho internacional humanitario y los tratados internacionales de los que fuera parte Camboya.

El Estatuto de constitución de estas Salas incluyen entre los crímenes de derecho internacional humanitario por primera vez como una conducta punible independiente del tipo general de los crímenes de guerra la destrucción del patrimonio cultural de acuerdo con la Convención para la Protección de los Bienes Culturales en caso de Conflicto Armado de $1954^{27}$.

Esta novedosa previsión inicia la posibilidad de perseguir tales actos de forma independiente y sin necesidad de ligarla a la comisión de cualquier otro acto de crimen de guerra. Pero de forma más notable, extiende la protección de los bienes a un conflicto que únicamente podría ser calificado como interno en virtud de la legislación internacional vigente en ese momento, y en el que las violaciones graves del patrimonio cultural solo podrían perseguirse en base al derecho interno.

El régimen de la Kampuchea Democrática desató un genocidio sin precedentes ${ }^{28}$, eliminando a un $20 \%$ de la población total de Camboya en ese momento en un período de cuatro años. Así se ha determinado recientemente por las Salas Extraordinarias, en su sentencia de 18 de noviembre del presente año, en el asunto 002/02 contra los máximos líderes políticos del régimen de los khemeres rojos que siguen con vida. Junto con las prácticas genocidas, el régimen de los khemeres eliminó los símbolos culturales que se consideraban contrarios al pensamiento comunista. Está por comprobar si las Salas llegan a incluir este tipo penal en alguna de las actas de acusación, ya que por el momento este no ha sido el caso.

\section{Conclusión.}

Como hemos podido analizar, es cierto que la labor de persecución penal internacional de las destrucciones del patrimonio cultural parece estar teñida de cierta priorización entre los crímenes, persiguiéndose únicamente los casos en los que la destrucción del patrimonio va ligada a otros crímenes contra las personas. Sin embargo, tal labor debería ser corregida, ya que como afirmaba la Relatora Especial sobre los derechos humanos, Karima Bennoune:

\footnotetext{
${ }^{27}$ Según establece el artículo 7 del Estatuto de las Salas, The Extraordinary Chambers shall have the power to bring to trial all Suspects most responsible for the destruction of cultural property during armed conflict pursuant to the 1954 Hague Convention for Protection of Cultural Property in the Event of Armed Conflict, and which were committed during the period from 17 April 1975 to 6 January 1979 ".

28 Sobre el genocidio en Camboya, véase: WILLIAMS, S., "Genocide: The Cambodian experience", International Criminal Law Review, 5(3), 2005, pp. 447-462.
}

CEED Número 39 Publicación actas Congreso Internacional 70 Aniversario Declaración Universal de Derechos 
"el derecho al acceso y al disfrute del patrimonio cultural forma parte del derecho internacional de los derechos humanos. El patrimonio cultural está vinculado con la dignidad y con la identidad humanas. Además, si bien algunos aspectos concretos del patrimonio material pueden tener especial repercusión para determinados grupos de personas, o guardar con ellos un vínculo particular, toda la humanidad tiene lazos con esos bienes, que representan "el patrimonio cultural de toda la humanidad". Por lo tanto, todos los Estados tienen la obligación de respetar y proteger el patrimonio cultural de conformidad con las normas internacionales, garantizar la rendición de cuentas respecto de actos de destrucción intencional de ese patrimonio, y cooperar para protegerlo" 29.

Ante la limitada jurisdicción de la CPI a este respecto y en relación con la persecución, los Estados, en cumplimiento de su obligación de proteger, serán los que deban extender su jurisdicción sobre los crímenes contra el patrimonio cultural, cumpliendo con sus obligaciones internacionales y participando en el desarrollo y protección de los derechos culturales.

${ }^{29}$ Vide nota 1, párr. 89.

CEED Número 39 Publicación actas Congreso Internacional 70 Aniversario Declaración Universal de Derechos 
GERTENBLITH, P., "Archaeology in the Context of War: Legal Frameworks for Protecting Cultural Heritage during Armed Conflict”, Archaeologies: Journal of World Archaeological Congress, 5/1, 2009, pp. 18-31.

VAN DER AUWERA, S., "Contemporary Conflict, Nationalism, and the Destruction of Cultural Property during Armed Conflict: A Theoretical Framework, Journal of Conflict Archaeology, 7/1, 2012, pp. 49-65.

LOSTAL BECERRIL, M., La protección de bienes culturales en el Tribunal Internacional Penal para la Ex Yugoslavia, REEI, 24, 2012, 25 págs. http://www.reei.org/index.php/revista/num24/notas/proteccion-bienes-culturales-tribunalpenal-internacional-para-ex-yugoslavia

BADENES CASINO, M., La protección de los bienes culturales durante los conflictos armados. Especial referencia al conflicto armado en el territorio de la antigua Yugoslavia, Valencia, Universitat de València, 2005, 162 pp.

MERON, T., "The Protection of Cultural Property in the Event of Armed Conflict within the Case-law of the International Criminal Tribunal for the Former Yugoslavia”, Museum International, 57/4, 2005, pp. 41-60.

LAMBOURNE, W., "The Khmer Rouge Tribunal: Justice for Genocide in Cambodia?, en Law and Society Association Australia and New Zealand (LSAANZ) Conference, 'W(h)ither Human Rights', 2008, pp. 1-11.

KLEIN, K. M., "Bringing the Khmer Rouge to Justice: The Challenges and Risks Facing the Joint Tribunal in Cambodia", Northwestern University Journal of International Human Rights, 4(3), 2005, pp. 549-566.

WILLIAMS, S., “Genocide: The Cambodian experience”, International Criminal Law Review, 5(3), 2005, pp. 447-462.

CEFD Número 39 Publicación actas Congreso Internacional 70 Aniversario Declaración Universal de Derechos 